\title{
Electromagnetic modeling of a lightning rod
}

\author{
D. Poljak ${ }^{1}$, M. Birkic ${ }^{2}$, D. Kosor ${ }^{3}$, C. A. Brebbia ${ }^{4}$ \& V. Murko \\ ${ }^{1}$ Department of Electronics, University of Split, Croatia \\ ${ }^{2}$ Technical Department, Air Traffic Center Pula, Croatia \\ ${ }^{3}$ Technical Department, Air Traffic Center Split, Croatia \\ ${ }^{4}$ Wessex Institute of Technology, UK \\ ${ }^{5}$ Iskra Zascite, Slovenia
}

\begin{abstract}
This paper deals with a frequency domain analysis of a lightning rod using the antenna theory model. The lightning rod struck by lightning is represented by a straight thin wire antenna excited by an equivalent current source. The current induced along a lightning rod due to a direct lightning strike is determined by solving the homogeneous integro-differential equation of the Pocklington type. Once obtaining the current distribution along the rod provides the calculation of the charge induced along the rod and related irradiated electric field. The corresponding Pocklington equation and field integral relationships are handled via the Galerkin-Bubnov scheme of the Indirect Boundary Element Method (GBIBEM).
\end{abstract}

\section{Introduction}

Lightning flash surges are common sources of electromagnetic interferences (EMI) induced on electrical and electronic systems and equipment, thus producing many undesired effects, or even malfunction of systems and equipment [1-3]. The purpose of a lightning protection system (LPS) in terms of lightning rod is to capture a direct lightning strike. The main parameter of LPS is its efficiency which measure is related to the probability of direct strike to LPS instead of strike to the object within the protected volume defined by the protection zone. The protection zone is considered to be substantially immune to lightning strike due to the air terminal, i.e. the lightning rod. The protection zone around the rod can be assessed by applying the full wave analysis [2]. In particular, the protection zone can be determined if the electric field in the vicinity of the rod is known $[4,5]$. 
This paper first deals with the calculation of electric field around the rod using the antenna theory and boundary element analysis. The antenna model of the lightning rod used in this work is based on the homogeneous Pocklington integrodifferential equation [2,3] by which the current distribution along the rod is governed. The Pocklington equation is solved via the Galerkin-Bubnov variant of the indirect Boundary Element Method (GB-IBEM) [6].

Once the induced current along the rod is obtained one may calculate the related induced charge along the rod featuring the continuity equation and the irradiated electric field from the corresponding integral formulas using BEM formalism.

\section{Equivalent antenna model of the lightning rod}

The geometry of the problem is shown in Fig. 1. The lightning rod of length $L$ and radius $a$, sits vertically on a perfectly conducting (PEC) ground. According to the image theory the equivalent representation in terms of straight wire antenna is presented in Fig. 2. The wire is assumed to be perfectly conducting and its dimensions satisfy the thin wire approximation (TWA) conditions [2, 3].

The calculation of the induced current along the lightning rod due to the lightning return stroke is carried out by using the end-fed thin wire model [1-3], Fig. 2.

\subsection{Integral equation for a current distribution along the rod}

The homogeneous Pocklington integro-differential equation for the current distribution along the lightning rod can be derived by expressing the electric field in terms of the magnetic vector potential and by satisfying certain continuity conditions for the tangential field components at the PEC cylinder surface [5].

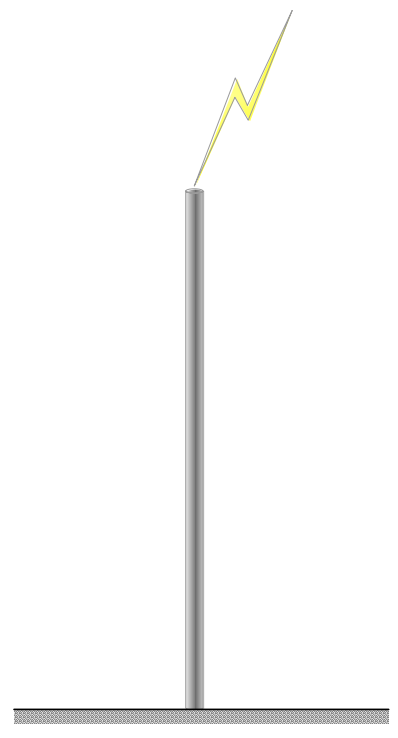

Figure 1: Lightning rod. 


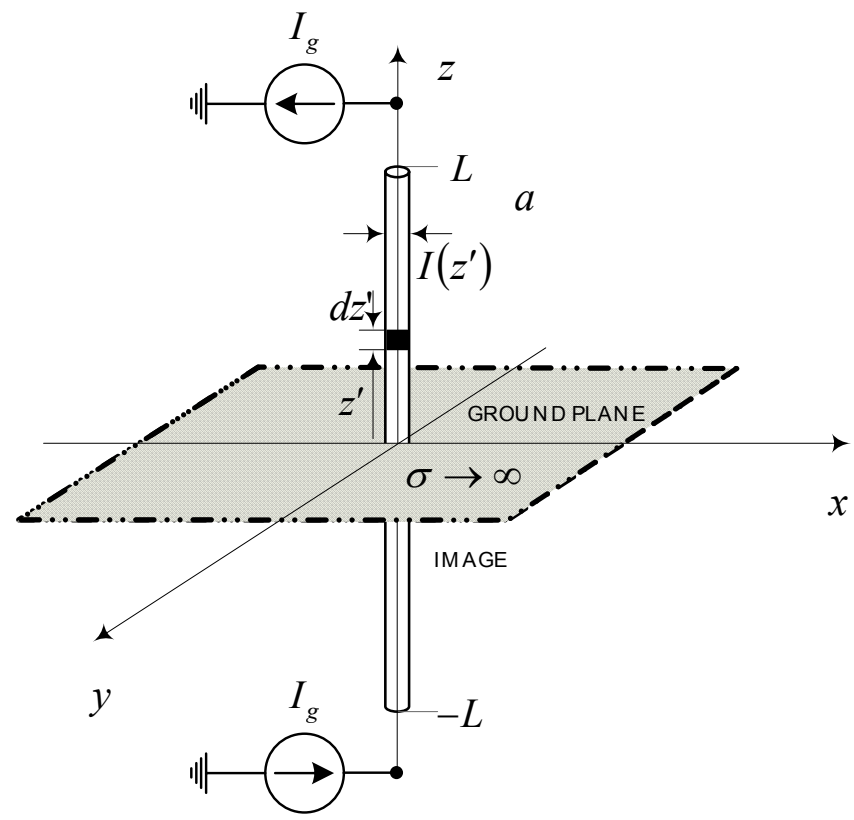

Figure 2: Equivalent antenna model of a lightning rod.

The electric field vector expressed in terms of magnetic vector potential [6], is given by:

$$
\vec{E}=\frac{1}{j \omega \mu \varepsilon_{0}} \nabla(\nabla \vec{A})-j \omega \vec{A}
$$

where $k$ is the phase constant of free space:

$$
k^{2}=\omega^{2} \mu_{0} \varepsilon_{0}
$$

while $\varepsilon_{0}$ and $\mu_{0}$ denotes the permitivity and permeability of the free space.

Due to rotational symmetry of the problem the radiated electric field does not depend on azimuth variable $\Phi$ and the electric field components are given by:

$$
\begin{gathered}
E_{\rho}=\frac{1}{j \omega \mu \varepsilon_{0}} \frac{\partial^{2} A_{z}}{\partial \rho \partial z} \\
E_{z}=\frac{1}{j \omega \mu \varepsilon_{0}} \frac{\partial^{2} A_{z}}{\partial z^{2}}-j \omega A_{z}
\end{gathered}
$$

The vector potential $z$-component is given by the particular integral [6]: 


$$
A_{z}=\frac{\mu}{4 \pi} \int_{-L}^{L} g\left(x, z, z^{\prime}\right) I\left(z^{\prime}\right) d z^{\prime}
$$

where $I\left(z^{\prime}\right)$ is the unknown current distribution along the rod, $g\left(\mathrm{x}, \mathrm{z}, \mathrm{z}^{\prime}\right)$ is the free space Green function of the form:

$$
g\left(x, z, z^{\prime}\right)=\frac{e^{-j k R}}{R}
$$

and $R$ is the distance from the source point on the rod to the arbitrary observation point in free space.

The total tangential electric field on the PEC wire surface $(\rho=a)$ vanishes, i.e. the interface condition is given by:

$$
E_{z}^{e x c}(a, z)+E_{z}^{s c t}(a, z)=0
$$

where $E_{z}^{e x c}$ is the excitation function and $E_{z}^{s c t}$ is the related scattered field.

Combining the relations (4) to (7) results in the Pocklington integro-differential equation for the unknown current:

$$
E_{z}^{e x c}=-\frac{1}{j 4 \pi \omega \varepsilon_{0}} \int_{-L}^{L}\left[\frac{\partial^{2}}{\partial z^{2}}+k^{2}\right] g_{a}\left(z, z^{\prime}\right) I\left(z^{\prime}\right) d z^{\prime}
$$

where $g_{a}$ is the integral equation kernel:

$$
g_{a}\left(x, z, z^{\prime}\right)=\frac{e^{-j k R}}{R}
$$

$R_{a}$ is the distance from the source point in the wire axis to the arbitrary observation point at the wire surface:

$$
\mathrm{R}_{\mathrm{a}}=\sqrt{\left(\mathrm{z}-\mathrm{z}^{\prime}\right)^{2}+a^{2}}
$$

As the excitation function is not available in the form of electric field, i.e. the equivalent antenna is neither driven by a voltage source, nor excited by a plane wave, the left-hand side of the equation (8) vanishes:

$$
-\frac{1}{j 4 \pi \omega \varepsilon_{0}} \int_{-L}^{L}\left[\frac{\partial^{2}}{\partial z^{2}}+k^{2}\right] g_{a}\left(z, z^{\prime}\right) I\left(z^{\prime}\right) d z^{\prime}=0
$$


As it is well-known from the antenna theory the integro-differential equation kernel becomes quasisingular [6] due to the presence of the second-order differential operator. This problem can be overcome by applying the so-called weak formulation of the problem and Galerkin Bubnov indirect Boundary Element Method (GB-IBEM). Thus, utilizing the property of the kernel

$$
\frac{\partial g_{a}\left(z, z^{\prime}\right)}{\partial z}=\frac{\partial g_{a}\left(z, z^{\prime}\right)}{\partial z^{\prime}}
$$

the alternative form of the integro-differential equation is obtained:

$$
-\frac{1}{j 4 \pi \omega \varepsilon_{0}}\left[\int_{-L}^{L} \frac{\partial I\left(z^{\prime}\right)}{\partial z^{\prime}} \frac{\partial g_{a}\left(z, z^{\prime}\right)}{\partial z} d z^{\prime}+k^{2} \int_{-L}^{L} I\left(z^{\prime}\right) g_{a}\left(z, z^{\prime}\right) d z^{\prime}\right]=0
$$

Solving the Pocklington equation the antenna current is obtained. The mathematical details on the BEM solution of equation (13) are presented in Appendix A.

\subsection{The boundary (end) conditions}

The equivalent antenna is excited by an ideal current generator with one terminal connected to the antenna and the other one grounded in the remote point in the space. This current source can be included into the integral equation scheme through the forced boundary condition applied at the top of rod. In accordance to the image theory the imaged current source is also injected on the top of the image rod, as shown in Fig.2.

Therefore, the applied symmetric boundary condition can be written, as follows:

$$
I(-L)=I(L)=I_{g}
$$

where $I_{g}$ denotes the equivalent current source.

The proposed model can be further upgraded by taking into account the influence of the lossy earth [6,7], and by including the lightning channel attached to the structure [1].

\subsection{The induced charge along the rod}

A deeper insight into the lightning phenomena and behaviour of LPS is provided by the assessment of the charge distribution induced along the cylinder rod struck by lightning. The linear charge density along the rod can be readily computed from the continuity equation [7], as follows:

$$
q(z)=-\frac{1}{j \omega} \frac{d I(z)}{d z}
$$

where $I(z)$ is the current distribution along the rod. 


\subsection{The electric field integral formulas}

The electric field irradiated by the equivalent antenna representing the lightning rod can be determined knowing the current distribution induced along the rod.

The radial (normal) field component can be determined inserting the expression for the magnetic vector potential (5) into equation (3):

$$
E_{\rho}=\frac{1}{j 4 \pi \omega_{\varepsilon_{0}}} \int_{-L}^{L} I\left(z^{\prime}\right) \frac{\partial^{2} g\left(z, z^{\prime}, \rho\right)}{\partial \rho \partial z} d z^{\prime}
$$

Performing the integration by parts equation (16) becomes:

$$
E_{\rho}=\frac{1}{j 4 \pi \omega \varepsilon_{0}} \int_{-L}^{L} \frac{\partial I\left(z^{\prime}\right)}{\partial z^{\prime}} \frac{\partial g\left(z, z^{\prime}, \rho\right)}{\partial \rho} d z^{\prime}
$$

The z-component of the electric field is defined by equations (4) and (5), i.e.:

$$
E_{z}=-\frac{1}{j 4 \pi \omega \varepsilon_{0}} \int_{-L}^{L}\left[\frac{\partial^{2}}{\partial z^{2}}+k^{2}\right] g_{0}\left(z, z^{\prime}, \rho\right) I\left(z^{\prime}\right) d z^{\prime}
$$

and after integration by parts it follows:

$$
E_{z}=-\frac{1}{j 4 \pi \omega \varepsilon_{0}}\left[\int_{-L}^{L} \frac{\partial I\left(z^{\prime}\right)}{\partial z^{\prime}} \frac{\partial g\left(z, z^{\prime}, \rho\right)}{\partial z} d z^{\prime}+k^{2} \int_{-L}^{L} I\left(z^{\prime}\right) g\left(z, z^{\prime}, \rho\right) d z^{\prime}\right]
$$

The integrals in expressions (17) to (19) contain quasi-singular kernel due to the presence of differential operator [6]. This quasi-singularity can be efficiently treated by the boundary element/finite differences approach [6, 7]. The mathematical details on computation of field components are given in Appendix B.

\section{Numerical results}

First computational example is related to the single lightning rod of length $L=10 \mathrm{~m}$ and radius $a=0.019 \mathrm{~m}$. Figures 3 and 4 show the induced current and charge along the rod excited by the unit current source at frequency $f=1 \mathrm{kHz}$. Figure 5 shows the tangential and the normal component of the irradiated field $\rho=10 \mathrm{~m}$ away from the rod. Figure 6 and 7 show the induced current and charge, respectively, along the rod with the same dimensions excited by the unit current source at the frequency $f=1 \mathrm{MHz}$, while Fig 8 shows the related tangential and normal field component.

The last set of Figs is related to the lightning rod protecting the radar antenna system of length $L=7.5 \mathrm{~m}$, radius $a=0.5 \mathrm{~m}$ excited by the unit current source at frequency $f=1 \mathrm{MHz}$.

Figs 9 and 10 show the normal and axial field component, respectively. 


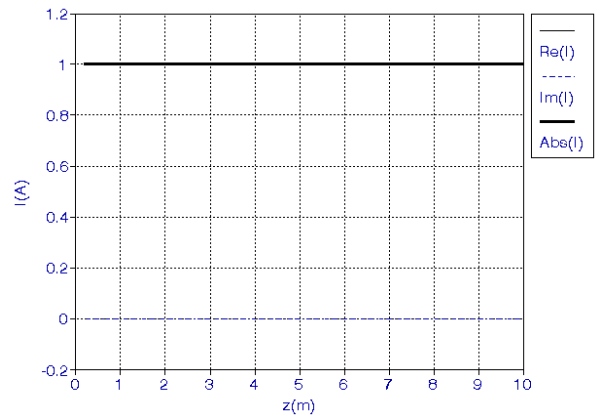

Figure 3: Real, imaginary and absolute value of the induced current $(L=10 \mathrm{~m}$, $a=0.019 \mathrm{~m}, f=1 \mathrm{kHz}$ ).

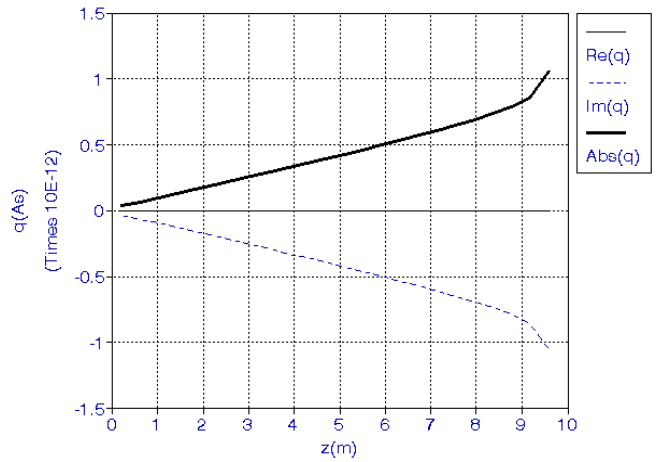

Figure 4: Real, imaginary and absolute value of the induced charge $(L=10 \mathrm{~m}$, $a=0.019 \mathrm{~m}, f=1 \mathrm{kHz})$.

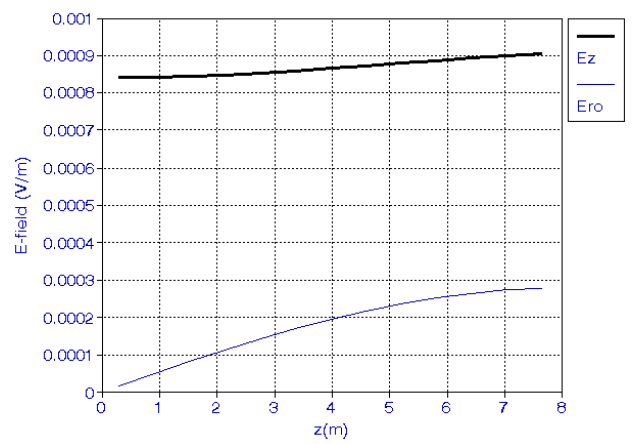

Figure 5: Absolute value of the tangential and normal field components $(L=10 \mathrm{~m}, a=0.019 \mathrm{~m}, f=1 \mathrm{kHz})$. 
286 Boundary Elements and Other Mesh Reduction Methods XXIX

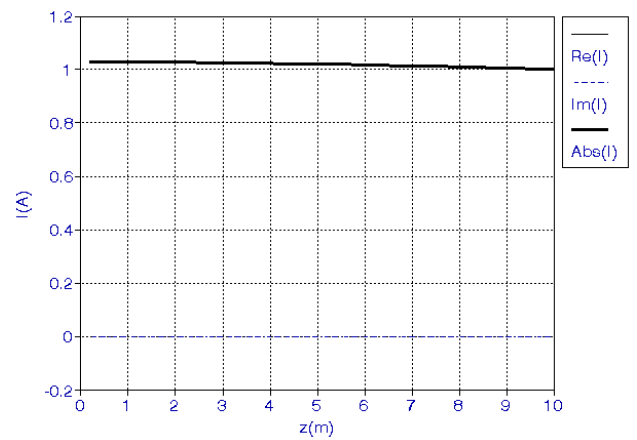

Figure 6: Real, imaginary and absolute value of the induced current $(L=10 \mathrm{~m}$, $a=0.019 \mathrm{~m}, f=1 \mathrm{MHz})$.

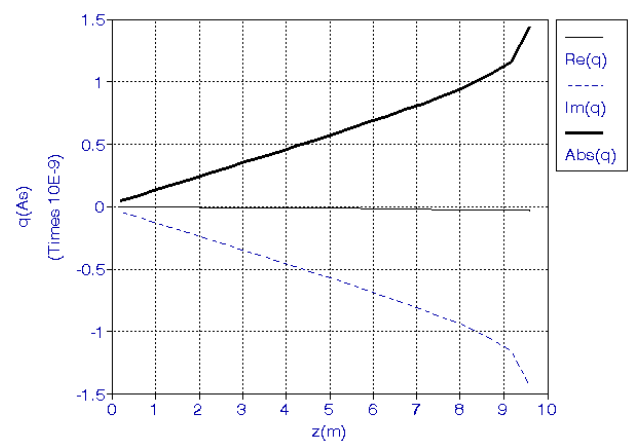

Figure 7: Real, imaginary and absolute value of the induced charge $(L=10 \mathrm{~m}$, $a=0.019 \mathrm{~m}, f=1 \mathrm{MHz})$.

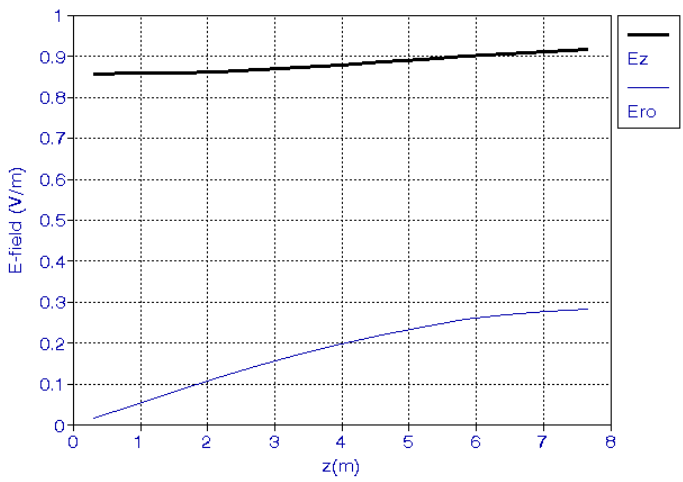

Figure 8: Absolute value of the tangential and normal field components $(L=10 \mathrm{~m}, a=0.019 \mathrm{~m}, f=1 \mathrm{MHz})$. 


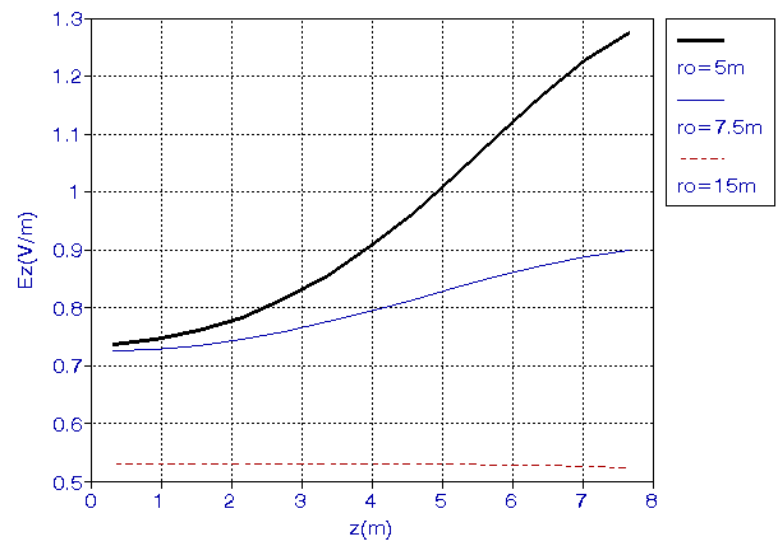

Figure 9: Absolute value of the tangential field component for different distances from the $\operatorname{rod}(L=7.5 \mathrm{~m}, a=0.5 \mathrm{~m}, f=1 \mathrm{MHz})$.

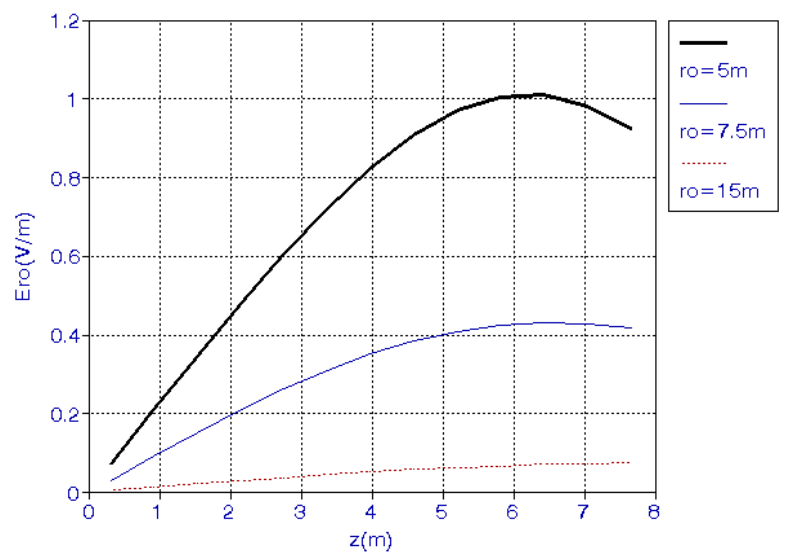

Figure 10: Absolute value of the normal field component for different distances from the $\operatorname{rod}(L=7.5 \mathrm{~m}, a=0.5 \mathrm{~m}, f=1 \mathrm{MHz})$.

\section{Concluding remarks}

The frequency domain analysis of the single lightning rod representing the simple lightning protection system (LPS) is undertaken in this work. The full wave model is based on the wire antenna theory. The lightning induced current along the rod is obtained as the BEM solution of the corresponding homogeneous integro-differential equation of the Pocklington type. Having obtained the current distribution it is possible to compute the induced charge along the rod and the related irradiated electric field. Further extension of the present analysis will involve the treatment of complex lightning protection systems consisting of conductors in vertical and horizontal arrangement. A particular feature of the future work will be related to the direct time domain analysis of the problem. 


\section{Appendix A: BEM solution of homogeneous Pocklington equation}

The Pocklington integro-differential equation (13) is numerically handled by means of the Galerkin-Bubnov scheme of the indirect Boundary Element Method [6].

The operator form of equation (13), can be symbolically written as:

$$
K(I)=0
$$

where $K$ is a linear operator and $I$ is the unknown current to be determined.

The unknown current is expressed by the sum of $n$ linearly independent basis functions $\left\{f_{i}\right\}$ with unknown complex coefficients $I_{i}$, i.e.:

$$
I \cong I_{n}=\sum_{i=1}^{n} I_{i} f_{i}
$$

Applying the weighted residual approach and choosing the test functions to be the same as basis functions (Galerkin-Bubnov procedure) the operator equation (A1) is transformed into a system of algebraic equations:

$$
\sum_{i=1}^{n} I_{i} \int_{0}^{L} K\left(f_{i}\right) f_{j} d z=0 \quad j=1,2, \ldots, n
$$

Performing certain mathematical manipulations the following matrix equation is obtained:

$$
\sum_{i=1}^{M}[Z]_{j i}\{I\}_{i}=0, \quad j=1,2, \ldots, M
$$

where the vector $\{\mathrm{I}\}$ contains the unknown coefficients, and $M$ is the total number of boundary elements.

The mutual impedance matrix $[\mathrm{Z}]_{\mathrm{ji}}$ representing the interaction of the $\mathrm{i}$-th source boundary element with the $\mathrm{j}$-th observation element is given by:

$$
[Z]_{j i}=-\frac{1}{j 4 \pi \omega \varepsilon}\left(\begin{array}{l}
\int_{z_{j}}^{z_{j+1}}\{D\}_{j} \int_{z_{i}}^{z_{i+1}}\{D\}_{i}^{T} g_{a}\left(z, z^{\prime}\right) d z^{\prime} d z+ \\
+k^{2} \int_{z_{j}}^{z_{j+1}}\{f\}_{j} \int_{z_{i}}^{z_{i+1}}\{f\}_{i}^{T} g_{a}\left(z, z^{\prime}\right) d z^{\prime} d z
\end{array}\right)
$$


where $\mathrm{z}_{i}, \mathrm{z}_{i+1}, z_{j}$ and $z_{j+1}$ are the coordinates of $i$-th and $j$-th wire segment, respectively.

A linear approximation over each boundary element has been used in this paper as this choice had already been shown to provide accurate and stable results $[6,7]$. Matrices $\{f\}$ and $\{f\}$ contain the linear shape functions:

$$
f_{i}(z)=\frac{z_{i+1}-z}{\Delta z} \quad f_{i+1}(z)=\frac{z-z_{i}}{\Delta z}
$$

while $\{D\}$ and $\left\{D^{\prime}\right\}$ contain their derivatives:

$$
\left.\frac{\partial I\left(z^{\prime}\right)}{\partial z}\right|_{z=z_{i}}=\frac{I_{i+1}-I_{i}}{\Delta z}
$$

\section{Appendix B: BEM evaluation of the field integrals}

The radial field component, defined by the integral:

$$
E_{\rho}=\frac{1}{j 4 \pi \omega_{\varepsilon_{0}}} \int_{-L}^{L} \frac{\partial I\left(z^{\prime}\right)}{\partial z^{\prime}} \frac{\partial g\left(z, z^{\prime}, \rho\right)}{\partial \rho} d z^{\prime}
$$

can be evaluated using the BEM formalism, i.e. the current along the segment can be written as:

$$
I(z)=I_{i} f_{i}(z)+I_{i+1} f_{i+1}(z)
$$

and, therefore, it follows:

$$
E_{x}=\frac{1}{j 4 \pi \omega_{\varepsilon_{0}}} \sum_{i=1}^{M} \frac{I_{i+1}-I_{i}}{\Delta z} \int_{z_{i}}^{z_{i+1}} \frac{\partial g\left(x, z, z^{\prime}\right)}{\partial x} d z^{\prime}
$$

Furthermore, to overcome the quasisingularity problem, the kernel is approximated via central finite difference formula:

$$
\frac{\partial f(x, y)}{\partial x}=\frac{f(x+\Delta x, y)-f(x-\Delta x, y)}{2 \Delta x}
$$

and the final expression for the radial electric field is then:

$$
E_{\rho}=\frac{1}{j 4 \pi \omega_{\varepsilon_{0}}} \sum_{i=1}^{M} \frac{I_{i+1}-I_{i}}{\Delta \rho \Delta z} \int_{z_{i}}^{z_{i+1}}\left[G\left(z, z^{\prime}, \rho+\Delta \rho\right)-G\left(z, z^{\prime}, \rho\right)\right] d z^{\prime}
$$

where $\Delta \rho$ denotes the finite difference step. 
The tangential field component is given by:

$$
E_{z}=-\frac{1}{j 4 \pi \omega_{\varepsilon_{0}}}\left[\int_{-L}^{L} \frac{\partial I\left(z^{\prime}\right)}{\partial z^{\prime}} \frac{\partial G\left(z, z^{\prime}, \rho\right)}{\partial z} d z^{\prime}+k^{2} \int_{-L}^{L} I\left(z^{\prime}\right) G\left(z, z^{\prime}, \rho\right) d z^{\prime}\right]
$$

Using linear interpolation for current over the segment yields:

$$
E_{z}=-\frac{1}{j 4 \pi \omega_{\varepsilon_{0}}} \sum_{i=1}^{M}\left\{\begin{array}{l}
\frac{I_{i+1}-I_{i}}{\Delta z} \int_{z_{i}}^{z_{i+1}} \frac{\partial G\left(z, z^{\prime}, \rho\right)}{\partial z} d z^{\prime}+ \\
k^{2} \int_{z_{i}}^{z_{i+1}}\left[I_{i} f_{i}(z)+I_{i+1} f_{i+1}(z)\right] G\left(z, z^{\prime}, \rho\right) d z^{\prime}
\end{array}\right\}
$$

and approximating the kernel with finite differences the final formula for the axial field component is:

$$
E_{z}=-\frac{1}{j 4 \pi \omega_{\mathcal{E}_{0}}} \sum_{i=1}^{M} \frac{I_{i+1}-I_{i}}{\Delta z^{2}}\left\{\begin{array}{l}
\int_{z_{i}}^{z_{i+1}}\left[G\left(z+\Delta z / 2, z^{\prime}, \rho\right)-G\left(z-\Delta z / 2, z^{\prime}, \rho\right)\right] d z^{\prime} \\
+k^{2} \int_{z_{i}}^{z_{i+1}}\left[I_{i} f_{i}(z)+I_{i+1} f_{i+1}(z)\right] G\left(z, z^{\prime}, \rho\right) d z^{\prime}
\end{array}\right\}
$$

\section{References}

[1] F.C. Yang, K.S.H. Lee, Natural Frequencies of a Rod with a Lightning Return Stroke, pp. 75-86, in Lightning Electromagnetics, R. Gardner, (Ed) CRC Press 1990.

[2] D. Poljak, B. Jajac, "Lightning Induced Current on a Metallic RodFrequency Domain Analysis " ICECOM '97, pp 87-90 Dubrovnik, Croatia, Oct. 1997.

[3] D. Poljak, B. Jajac, "On the Use of Monopole Antenna Model in Lightning Protection System Analysis " EMC'98 ROMA, pp 370-374, Roma, Italy, Sept. 1998.

[4] K. Aniserowitz, "Methods of Creation of Lightning Protection Zones Near Tall Telecommunication Structures According to Different National Standards ", TCSET' 2002, Lviv- Slavsko, Ukraine, Feb 18-23, 2002.

[5] B. Jajac, D. Poljak, N. Kovac "Boundary Element Modelling of the Metallic Rod Protection Zone", BEM XXV, Southampton, UK, Boston, and USA: WIT Press, 2003.

[6] D. Poljak, C.A. Brebbia, "Boundary Element methods for Electrical Engineers ", WIT Press, Southampton-Boston, 2005.

[7] D. Poljak, V. Doric, S. Antonijevic "Computer aided Design of Wire Structures: Frequency and Time Domain Analysis", WIT Press, Southampton-Boston, 2007. 